\title{
Imaging, Flow Cytometry, and Functional Cytomics.
}

\author{
J. Paul Robinson, $\mathrm{PhD}$ \\ Purdue University Cytometry Laboratories, Purdue University, West Lafayette, IN 47907 \\ www.cyto.purdue.edu
}

This presentation will discuss how the next generation of cell analysis technologies will interact to provide insight into the complex pathways that involve mechanisms for transcription, translation, and metabolic or signal transduction systems. In the past, most people have focused on individual components of these pathways by developing individual systems with limited overlap. With the advent of high-throughput proteomics, the opportunity to study the inter-relationships between these pathways is very attractive. However, making sense of these complex environments requires a systems approach that incorporates multiple technologies from which common problems are studied. This then is where the potential of cytomics approaches may create new opportunities. These opportunities integrate a variety of experimental approaches can be applied to derive models of biochemical pathways and to identify the functions of novel genes. Thus, the endpoint from a cytomics perspective is to understand function at the cellular level.

Cytomics has been defined as the multiparameter cytometric analysis of the cellular heterogeneity of cytomes (cellular systems/organs/body), which utilize a maximum of information on the apparent molecular cell phenotypes, resulting from cell genotypes and exposure ${ }^{1 ; 2}$. Based on Valet's definition, cellular phenotypes in naturally existing cellular and cell population heterogeneity of disease-affected body cytomes contain the information on the future development (predictive) as well as on the present status (diagnosis) of a disease. Thus combining technologies that evaluate cellular and proteomics systems simultaneously have tremendous potential for diagnostics.

Functional genomics focuses on high-throughput analysis of the function of hundreds or thousands of genes at once. This bring a significant problem in data analysis and requires the development of software tools to handle and interpret the huge data sets, which is bioinformatics. In essence, biological processes are determined by the genome, revealed by the transcriptome, controlled and functionalized via the proteome, and enacted by the cell. The cytome is therefore a desirable endpoint for which technologies such as flow cytometry and imaging are highly suited.

The types of technologies that will be developed to participate in the cytomics revolution will be complex. For the most part, they will combine optical cellular bio-analysis systems that will expand current technologies using semiconductor chips in the continuing expansion of the importance of micro and nanotechnologies. For example, current development in the area of femto and picosecond excitation systems, nanoscale, optoelectronics and biocontrol systems will provide needed microscale detection systems.

Cytomics will be important because in the final analysis, it is the cell that is the ultimate functional endpoint. The cell is the minimal functional unit within our physiology and as such it is the functional unit that can be manipulated. The complexity of cell function is only part of why Cytomics will become a major field of study. Every cell is different. By studying each cell's unique function one can model that cell type for subsequent analysis. As the field of tissue engineering explodes, it will not be long before cellular engineering will be a most important component of which an essential element will be a full understanding of Cytomics.

Within a few years, no pharmaceutical company will operate without encompassing the essential features of Cytomics - they will be designing drugs that will operate at the level of modified cellular functions, cytomealignment or cytomic-realignment will become the "cellular form" of tissue engineering.

This knowledge will require a better-than-ever understanding of how the cell operates, how to measure cell function, and how to characterize the cell in minute detail. Single-cell analysis techniques will become 
enhanced and exquisitely sensitive. New technologies must be developed and new analytical tools will be required to extract these new data. Of these analytical tools, informatics will continue to play a crucial role in cell biology.

In the past decade, several important technologies have created a vast change in biomedical knowledge and practice. Many of these technologies have been at the molecular to the gene level. Biomedical techniques such as imaging modalities have been expanding tremendously. As genomics moves to proteomics and subsequently functional genomics, the issue at hand will be how do we better understand the involvement of the cell? To answer this question, scientists and biomedical engineers will need to work with cells in a way that evaluates function - not just composition or product formation. This then is in essence where Cytomics will play a major part.

We have several important cellular technologies in our investigative armament. Flow cytometry is the most effective single-cell analysis technology available today. In the past several years, high-speed sorters have been developed that can sort at 100,000 cells per second. High-throughput systems are currently being developed using flow principles that will be able to evaluate a million cell assays per day on a single instrument. Each cell will be characterized with at least 5-10 but possibly as many as 30-40 parameters. Alternative technologies using multiplexing offer even great capabilities. Currently it is predicted that 100 to 1000 measurements can be made per tube, running at 100 tubes per minute. The data collection and management tasks alone are enormous. In addition to all this, cellular and molecular imaging is expanding tremendously. Almost all the new molecular gene systems are image driven. Thus, fluorescence and imaging modalities will become more significant. These types of assay systems will drive the field of Cytomics, which is surely going to be a crucial field linking cell biology and biomedical engineering.

As we expand our detection tools we automatically expand the data capacity. Thus, managing these data will be a major challenge. Bioinformatics needs have already outstripped available technologies. The huge explosion in data-collection capability, resulting from the increased ease and speed with which computer-based instruments can collect data is a driving force in cytomics. The next several years will bring real opportunities to develop analytical skills in data processing and analysis. This will require massive databases and programs to develop correlated data sets.

Within this presentation, there will be a number of systems ranging from bacterial cultures in 3D biofilms to epithelial cultures in extracellular matrix, all of which require multiple technologies to understand the nature of the interactions within these systems.

\section{References}

1. Valet, G. Cytomics. 2003.http://www.biochem.mpg.de/valet/cytomics.html, Electronic Citation

2. Robinson, J. P. cytomics. www.cytomics.info, Electronic Citation 